\title{
ARDUINO BASED RADAR SYSTEM
}

Sarmad Hameed

Mechatronics department, Shaheed Zulfikar Ali Bhutto Institute of Science and Technology, Karachi, (Pakistan) E-mail: sarmad.hameed@szabist.edu.pk

Naqi Jafri Mechatronics department, Shaheed Zulfikar Ali Bhutto Institute of Science and Technology, Karachi, (Pakistan) E-mail: Naqijafri10@gmail.com

Dania Rashid

Mechatronics department, Shaheed Zulfikar Ali Bhutto Institute of Science and Technology, Karachi, (Pakistan) E-mail: daniarashid118@gmail.com

Fabiha Shoaib

Mechatronics department, Shaheed Zulfikar Ali Bhutto Institute of Science and Technology, Karachi, (Pakistan) E-mail: fabihashoaib@hotmail.com 


\section{ABSTRACT}

This paper is about Radar System controlled via Arduino. This RADAR system consists of an ultra-sonic sensor and servo motor, these are the major components of the system. Basic working of the system is that it have to detect objects in its defined range. Ultra-sonic sensor is attached to the servo motor it rotates about 180 degree and gives visual representation on the software called processing IDE. Processing IDE gives graphical representation and it also gives angle or position of the object and distance of the object. This system is controlled through Arduino. Arduino UNO board is sufficed to control ultrasonic sensor and also to interface the sensor and display device. While researching, we learned about existing navigation and obstacle detection innovations and different systems where ultrasonic sensors are used efficiently. Main application of this RADAR system comes into different field of navigation, positioning, object identification, mapping, spying or tracking and different applications. These less investment system are also suitable for indoor applications.

\section{KEYWORDS}

Arduino, ultra-sonic, radar, positioning, surveillance, obstacle detection.

\section{INTRODUCTION}

RADAR system is an object detection or tracking system which uses radio waves to decide or get the range, height, heading, or speed of items or objects. Radar frameworks or system arrive in an assortment of sizes and have distinctive performance particulars. Some radars are utilized for aviation authority at air terminals and others are utilized for long range observation and earlycautioning frameworks [1]. There are some ways to show radar working data. There are also some modified radar systems which have advance technology of handling the systems. These modified system are used at higher levels to get or extract the helpful or important data [2].

Our proposed system's working principle is linked by the following components which are is ultra-sonic sensor connected to the microcontroller (we have chosen Arduino) digital input and output pins. Then we have servo motor which is also connected to digital output and input pins. Our both main components ultra-sonic sensor and servo motor are connected simultaneously, so that when our servo motor rotates from 0 degree to 180 degree from extreme right to extreme left the motor will rotate nearby its axis [3]. We utilize Computer screen to demonstrate the data (distance and angle) through software called "Processing development Environment" [1].

\section{LITERATURE REVIEW}

Subsequent to experiencing a portion of the papers with respect to usage utilizing ultrasonic sensors and ARDUINO, it was found that this idea is searched a lot and is a mainstream idea which is still in advance. The advances utilized were not just productive and solid yet in addition financially achievable 
[5]. Not only this, here other very useful applications of ultrasonic sensors were observed too.

This paper discusses about a monitoring system which is designed measure to speed of waves and height of river through ultra-sonic sensor using microcontroller (Arduino). On the off chance that the waterway can't oblige the volume of water, then all the water will submerge with land and this phenomenon is called as flood or surge. We can overcome this flood problem by earlier identification in height of water and observing speed. If we identify problem earlier we can overcome this problem before it become crisis. By testing the system i.e. simple water level, it was observed that ultra-sonic have accuracy of $96.6 \%$. But when it is implemented in the rivers there are many errors because of different type of water levels due to heavy waves and speed of water and also due to floating of heavy objects. Unlike Previous testing results, author directed this analysis on tracking of speed of water improvement or modification and level of water in flooding. The test was completed when the Arduino used as controller of application. For more research, information of depth level and speed of water of this system will be sent to database server website to be checked regularly [8].

An intelligent driver monitoring and vehicle control system is introduced in this research. This technology is create to avoid accidents by monitoring the driver's activities. The writer states some of the main reasons of accidents today. These are alcohol consumption by the driver, carelessness, drowsiness or medical illness. The various units in the framework, including motors, relays, power unit and ESP8299 module are tried and are observed to be in working condition. Ultrasonic sensor is utilized to alarm the driver if any vehicle draws close to his vehicle. The status of the driver can be observed by the assistance of sensors executed in the vehicle and the subtle elements are refreshed to the proprietor. This system overcomes all the different aspects due to which other technologies designed for this purpose have failed, making the system more useful, efficient and less costly and less time consuming [7]. In this research paper authors have given information about the detection of radio waves and tracking or ranging through radar set which is built from components like an ultra-sonic sensor, a servo motor and an Arduino. The author discuss about the linear measurement problem because of which distance measurement was not possible between some objects, was resolved with the introduction of Ultrasonic distance measurer. It allows to take noncontact measurements. This radar system can drastically reduce power consumption. The author says, that this system is an extremely handy radar system, it can read or track the distance and angle of an obstacle and shown it up on the monitor screen. The ultra-sonic was attached on top of the servomotor to detect obstacles at 0 degree to 180 degree from right to left. Both the ultra-sonic sensor and the servo were fueled and controlled by the Arduino controller. The GUI was built using the JAVA programming language to show the result on the monitor [3].

This paper represents a system for obstacle detection in a known environment. This system works through an android based mobile camera. People who are 
visually impaired, face difficulties in detecting obstacles and navigation while they walk. They use sticks for this problem nonetheless this manner or technique is not right way of doing it. Object indicator or detector can overcome accidents or collision problems of people or the other way is they can to accurate map reading. The algorithm which is proposed in this paper is made for indoor mapping. In indoor surrounding all distinctive floors are taken in consideration and single image is kept or stored for distinctive floors. These images of floor are taken as reference image. The author mentions that this algorithm is $96 \%$ accurate and works in real time. There are different techniques discussed in this paper for obstacle detection. For these types of problems we can use the approach of SONAR sensor and also laser camera .In this paper introduced a calculation for identifying hindrance in known condition with an android based versatile camera which scans chosen territory before the camera for impediment location [9].

This research is about a blind walking stick made for blind people through which they can avoid obstacles while they walk and recognize currency. With the thought of visually impaired individuals, it is to some degree troublesome job to distinguish the cash or any unexpected obstacle. Despite the fact that currency dependent on size could possibly be recognized however it is relatively hard to distinguish that whether the note is unique or phony. So to overcome this issue the authors have designed the Currency Recognition Blind Walking Stick. A lot of work is done on currency recognition and obstacle detection using advanced technologies like optical character recognition, SURF and pattern extraction through colors. But none of these systems had the feature for obstacle detection for blind people. Therefore this framework is efficient as the other ones having an extra feature for helping the visually impaired [10].

\section{METHODOLOGY}

In order to testify the working of this system, after its designing, construction and programming we placed few objects in front of the ultrasonic sensor. As the motor started to rotate, our monitor started to display the output through processing IDE. Hence, when the sensor crossed over the object it showed a red segment with the distance and angle where the object is paced.

The first object was placed at the distance of $30.5 \mathrm{~cm}$ measured through a ruler and the system measured the distance at $32 \mathrm{~cm}$. While the second object was placed at a distance of $20 \mathrm{~cm}$ and the system measured it as $21 \mathrm{~cm}$. Hence the calculated efficiency turned out to be $95 \%$.

\section{System Overview:}




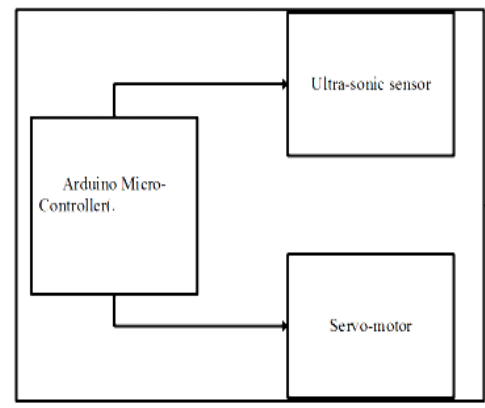

Figure 1. System hardware description.

The above figure represents a brief overview of this radar system. Here, as it is shown the controller we are using is Arduino, with the input Ultrasonic sensor and the output is the servo motor which rotates 180 degrees. The microcontroller controls all the operations of this system, from rotation of the motors to the obstacle detection of the ultrasonic and representation of the result on the screen.

\section{System Block Diagram:}

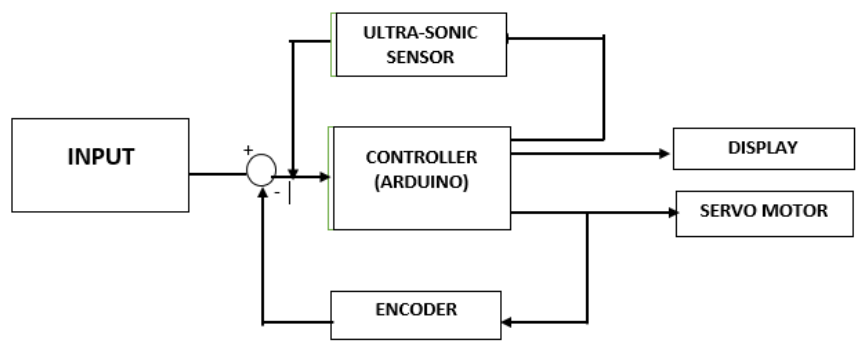

Figure 2. Block Diagram of Radar System.

Figure 2 represents the system's block diagram.

Here, it can be seen how the work flow in this radar system. The sensor is going to sense the obstacle and determine the angle of incident and its distance from the radar. The servo motor is constantly rotating to and fro, hence making the sensor move. The data obtained is encoded and fed to the processing IDE which represents it on the screen. The results are displayed further in this paper. All these operation are done by Arduino microcontroller from the rotation of the servo, data collection from the sensor, feeding the data to encoder to transferring it to the display.

\section{WORKING}

The basic objective of our design is to ascertain the distance position and speed of the obstacle set at some distance from the sensor. Ultrasonic sensor sends the ultrasonic wave in various ways by rotating with help of servo motors. This wave goes in air and gets reflected back subsequent to striking some object. This wave is again detected by the sensor and its qualities is analyzed and output is shown in screen indicating parameters, for example, distance and position of object. Arduino IDE is utilized to compose code and transfer coding in Arduino and causes us to detect position or angle of servo motor and it is communicated through the serial port alongside the covered distance of the nearest object in its way. Output of all of this working is shown in the software 
called processing, it will display the input/output and the range of the object [4]. Implementations of the sensors are done in such a way that ultra-sonic sensor is attached on top of the servo motor because it have to detect the object and its distance. Arduino (micro-controller) will control the ultra-sonic sensor and servo motor and also powered will be given to both of them through micro-controller [3].

Figure 3. Flow chart of Radar System.

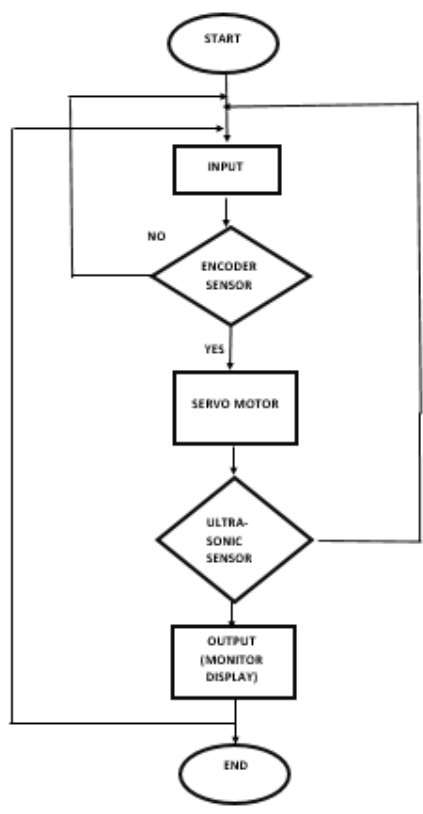

The above flow chart explains the working and the decision flow of this framework. As it can be seen the system starts with an input i.e. when the ultrasonic sensor detects an object, or does not detects any object, at any condition the encoder feeds the information in the controller while the servo keeps constantly rotating. As soon as any obstacle/objet is detected by the ultrasonic sensor the data is immediately processed by the controller and is fed to the IDE which shows it on the display screen. Here the process ends with an estimated distance of the object from the system with the angle at which it is placed.

\section{DISCUSSION AND RESULT}

In this research paper we have mentioned that our system is designed consisting following components such as, a servo- motor, an ultra-sonic sensor and a micro-controller (Arduino). System's objective is to track the distance and angle of the object and to represent this information graphically, means its output should be in graphical form which will be represented through processing software. We can have an idea of an efficiency of this radar by testing objects at different levels and observe how faster or smoothly it detects an object that it finds in a way and gives us an expected range of the obstacle [3].

Following figure show the results of the monitor screen of our design when the sensor rotates through the area and detects obstacle in the way. The red area indicates the presence of obstacle and below the angle of incident and distance is being displayed. 


\section{Testing of the system}

a) Object 1 is placed 30.5 far from the radar, radar gives the distance 32 $\mathrm{cm}$, so:

$$
\begin{array}{ll}
\circ & \text { error }=(32-30.5) / 30.5) * 100=4.918 \% \\
\circ & \text { efficiency } 1=100 \text {-error }=95.08 \%
\end{array}
$$

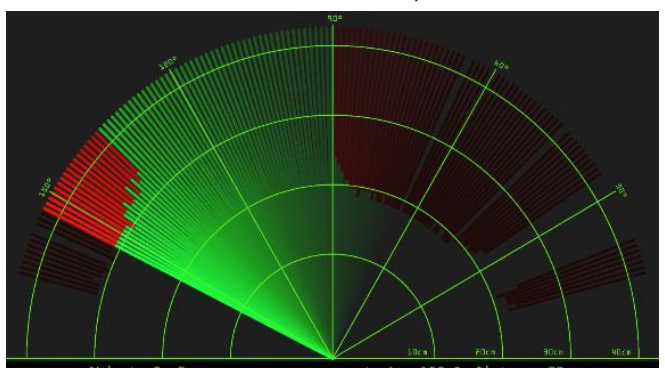

Figure 4. Processing IDE Screen displaying output of the system which we teseted by placing objects.

b) object 2 placed at a distance of $20.3 \mathrm{~cm}$,radar gives the distance $21 \mathrm{~cm}$ so:

○ $\quad$ error $=((21-20.3) / 20.3) * 100=3.44 \%$

○ efficiency $2=100$-error $=96.55 \%$

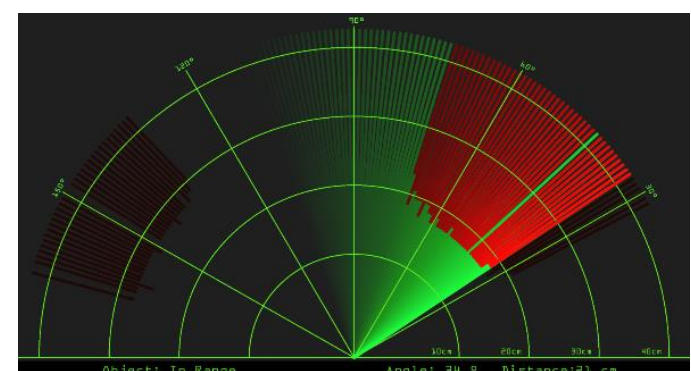

Figure 5. Processing IDE Screen displaying output of the system which we teseted by placing objects.

After the observations and calculations we can conclude that this system is $95.815 \%$ efficient.

\section{APPLICATION}

This Radar System have various applications for security purposes and it is mainly used for mapping.

- APPLCATION IN AIR FORCE:

It is used in airplanes or aircraft machines which have implemented radar system in it to detect the objects that comes in a way. It is also used to calculate height readings.

- APPLICATION IN MARINE:

This radar system also used in ships or marine. It is implemented on big ships to calculate the distance of other boats or ships, with the help of this sea accidents can also be reduced by not colliding. It can also be implemented on ports to see the distance of other ships and to monitor or control the ship movements.

- APPLICATON IN METEROLOGY: 
Meteorologists also uses radar systems to track or monitor the wind. It has been become an important equipment for climate testing. For example to detect tornados, storms.

\section{CONCLUSION}

Numerous advanced control methods gave designers to have more command over different advanced applications. In our paper, the recommended mapping method of whole system is assessed on small principles or scale [11]. The field that we have chosen for our design "Radar System" is a very vast field and future scope of this technology is very high. We have tremendous applications in which radar system have been implemented or used [3]. There is a lot of future scope of this design because of its security capacity. It can be used in many applications. This framework can also be developed or modified according to the rising needs and demand [4].

As we have designed a short range radar therefore our research was specified and limited. This system can only detect objects from 0 to 180 degrees only because the servo motor that we have used can rotate only to this range. So, due to this limitation our design cannot be applied to places or areas for obstacle detection on a larger scale. Usage of a 360 degrees rotating servo motor can make the system more efficient. We look forward to modify this system and enhance our research work by using a fully 360 degrees rotating servo and a higher ranged ultrasonic sensor. We can further add features to this system i.e. making it mobile, mounting an alarm system to it which turns on when obstacle is detected. Further modifications could be an obstacle avoiding robot with surveillance system.

\section{REFERENCES}

[1] Ahman Emmanuel Onoja, Abdusalaam Maryam Oluwadamilola, Lukman Adewale AJAO-"Embedded System Based Radio Detection and Ranging (RADAR) System Using Arduino and Ultra-Sonic Sensor” American Journal of Embedded Systems and Applications 2017

[2] Shreyes Mehta, Shashank Tiwari-"RADAR SYSTEM USING ARDUINO AND ULTRASONIC SENSOR” IJNRD, Volume 3, Issue 4 April 2018

[3] Antonio Tedeschi ; Stefano Calcaterra , Francesco Benedetto“ Ultrasonic RAdar System (URAS): Arduino and Virtual Reality for a Light-Free Mapping of Indoor Environments” IEEE Sensors Journal Volume: 17 , Issue: 14 , July 15, 152017

[4] Kiruthikamani.G, Saranya.B, Pandiyan.P-“Intelligent Driver Monitoring and Vehicle Control System” IJSRD - International Journal for Scientific Research \& Development| Vol. 5, Issue 09, 2017

[5] Mohanad Mahdi Abdulkareem,Qusay Adil Mohammed ,Muhanned Mahmood Shakir-“A Short Range Radar System"Rangefinder”,

[6] Harshad D. Lajurkar, Rushikesh D. Malokar, Akash N. Karmore“ Currency Recognition Blind Walking Stick” IJIRST -International 
Journal for Innovative Research in Science \& Technology,Volume 4 ,Issue 7 ,December 2017

[7] Srijan Dubey,Supragya Tiwari, Simit Roy -“ IMPLEMENTATION OF RADAR USING ULTRASONIC SENSOR” Indian J.Sci.Res. 2017

[8] Anuj Dutt (Author), 2014, Arduino based RADAR System, Munich, GRIN Verlag.

[9] T H Nasution, E C Siagian, K Tanjung, Soeharwinto-"Design of river height and speed monitoring system by using Arduino" 10th International Conference Numerical Analysis in Engineering 2018

[10] Syed M Taha Saquib, Sarmad Hameed, Syed M Usman Ali, Raza Jafri, Imran Amin-"Wireless Control of Miniaturized Mobile Vehicle for Indoor Surveillance" ICSICCST 2013 IOP Conf. Series: Materials Science and Engineering

[11] Sami ur Rahman, Sana Ullah and Sehat Ullah-" Obstacle Detection in Indoor Environment for Visually Impaired Using Mobile Camera"CCISP 7017, IOP Conf. Series: Journal of Physics: Conf. Series 2018. 


\section{AUTHORS}

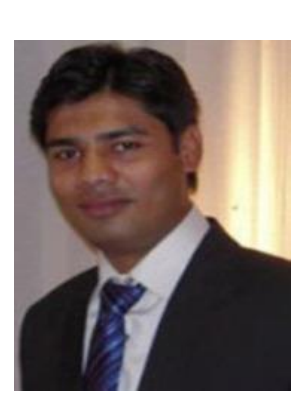

\section{Sarmad Hameed}

Sarmad Hameed is serving as Lecturer in Mechatronic Engineering Department at SZABIST. He has done his master degree in Industrial Controls and Automation Program from Hamdard University. He has earned his bachelor degree in Electronics from Sir Syed University of Engineering and Technology in 2010. His areas of interest are digital circuit designing, programming; troubleshooting, and industrial control systems design. His job description includes integration of renewable energy technologies into the electric power system.

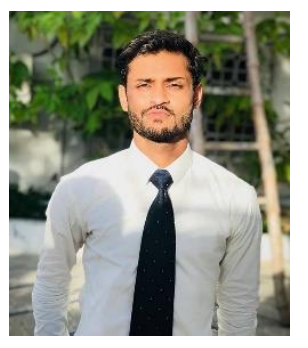

\section{Naqi Jafri}

Naqi Jafri currently enrolled in final year of B.E Mechatronics at SZABIST. His area of interest are IOT based system, robotics and embedded systems.

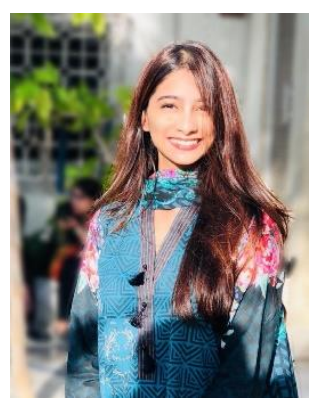

\section{Dania Rashid}

Dania Rashid is a final year student of Mechatronics Engineering department doing from SZABIST. Her area of interest are robot navigation, IOT based system and Simultaneous Localization and Mapping (SLAM).

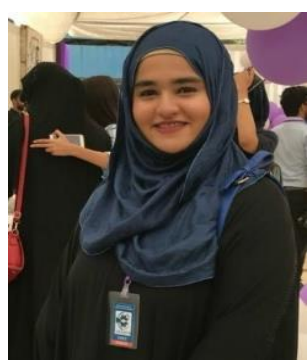

\section{Fabiha Shoaib}

Fabiha Shoaib currently enrolled in final year of B.E Mechatronics at SZABIST. Her field of interests are Autonomous \& Connected. 